\title{
Sperm parameters of honeybee drones exposed to imidacloprid
}

\author{
Andrzej Ciereszko ${ }^{1}$, Jerzy Wilde ${ }^{2}$, Grzegorz J. Dietrich ${ }^{1}$, Maciej SiUdA ${ }^{2}$, Beata BąK ${ }^{2}$, \\ Sylwia JUDYCKA ${ }^{1}$, Halina KAROL ${ }^{1}$ \\ ${ }^{1}$ Department of Gamete and Embryo Biology, Institute of Animal Reproduction and Food Research, Polish Academy of \\ Sciences in Olsztyn, Tuwima 10, 10-748, Olsztyn, Poland \\ ${ }^{2}$ Department of Apiculture, Faculty of Animal Bioengineering, University of Warmia and Mazury in Olsztyn, Słoneczna \\ 48, 10-957, Olsztyn, Poland
}

Received 28 February 2016 - Revised 1 July 2016 - Accepted 5 August 2016

\begin{abstract}
The objective of this study was to evaluate the effects of chronical exposure of honeybee drones to environmental (5 ppb) and non-environmental concentration (200 ppb) of imidacloprid (IMD) on sperm concentration, motility, viability, and mitochondrial membrane potential measured in semen obtained from 180 drones originating from 18 colonies. The results demonstrate that IMD exposure did not affect sperm concentration; however, there were significant differences in concentration within colonies. IMD exposure was associated with reductions in sperm motility, which also varied within colonies. Statistically significant interactions between IMD exposure and colony were found for active mitochondria and sperm viability. Our results strongly suggest that neonicotinoids can negatively affect honeybee drone sperm quality. It is important to emphasize that IMD actions can be strongly modulated according to the colony.
\end{abstract}

\section{Apis mellifera / imidacloprid / spermatozoa / motility / viability}

\section{INTRODUCTION}

During the past two decades, neonicotinoids have been a major group of highly effective and widely used insecticides. Imidacloprid (IMD) is one of the most popular and extensively used neonicotinoid insecticides to date; in 2008, it was the world's largest selling insecticide and second largest selling pesticide (Simon-Delso et al. 2015). Neonicotinoid residues in the nectar and pollen of bee-attractive crops such as oilseed rape constitute the major exposure vehicle to pollinators, such as honeybees or bumblebees (Pohorecka et al. 2012; Cresswell 2014). Neonicotinoids exert an important influence on

Corresponding author: A. Ciereszko,

a.ciereszko@pan.olsztyn.pl

Manuscript Editor: David Tarpy non-target invertebrates, including honeybees as the most highly studied species in this respect (Goulson 2013). These effects can be lethal but very often include a broad range of important sublethal impacts characterized by non-linear and non-intuitive patterns due to the complex interplay of receptor binding and gene reprogramming effects (Pisa et al. 2015). Sublethal effects may not directly cause bee mortality and/or colony collapse but rather may become lethal in time and/or render colonies more sensitive to further stresses ultimately leading to colony collapse (van der Sluijs et al. 2013; Dively et al. 2015).

There is growing awareness of the knowledge gap regarding the potential effects of neonicotinoid insecticides on the reproduction of pollinators (Blacquière et al. 2012). Whitehorn et al. (2012) have observed an $85 \%$ reduction in the production of new bumblebee queens 
(Bombus terrestris) after exposure to fieldrealistic levels of the neonicotinoid imidacloprid. The latter suggests that the impact of imidacloprid on the reproduction of wild-bee colonies is likely to be widespread and significant. Sandrock et al. (2014a) have demonstrated that chronic neonicotinoid dietary exposure has severe detrimental effects on solitary red mason bee (Osmia bicornis ) reproductive output manifested by a $50 \%$ reduction in total offspring production and a significantly male-biased offspring sex ratio. Cresswell (2011) pointed out that most available data on IMD effects on honeybees originate from studies of adult worker bees, whereas colony health depends on the success of all life stages, including drones. It is well established that poorly inseminated queens is one of the key factors affecting honeybee colonies (Severson and Erickson, 1989). Recently, Williams et al. (2015) demonstrated that the reproductive system of honeybee queens exposed to neonicotinoid pesticides was severely affected morphologically and physiologically. To our knowledge, the effects of IMD on the male reproductive system of insects have not been studied. Such studies are also justified by recently published results demonstrating impairments of testicular function in IMD-treated vertebrates (Bal et al. 2012; Cardone 2015; LopezAntia et al. 2015; Lonare et al. 2015).

Testing the sperm quality of drones is important to understand the mechanism of biology of honeybee mating, including drone fitness, polyandry, and sperm competition (Ben Abdelkader et al. 2014). The usefulness of single tests is very limited for the evaluation of sperm quality in drones (Wegener et al. 2012). Therefore, a multiple testing approach is recommended in order to include different aspects of drone reproductive performance. Sperm concentration measurement is important to evaluate the efficacy and/or disturbances to spermatogenesis and to evaluate if sufficient numbers of spermatozoa are produced over the period from spermatogenesis to sexual maturity to fertilize queens and ensure the productivity of colonies (Rhodes et al. 2011). Sperm motility reflects both energetic efficacy and the quality of the motility apparatus; it is also important for sperm transfer to a queen's spermatheca (Ruttner and Koeniger 1971; Baer 2005). Viability staining reflects functional quality of membrane permeability and was found to be especially useful for the evaluation of sperm competition in insects, including honeybees (Hunter and Birkhead 2002; Tofilski et al. 2012). Measurements of mitochondrial membrane potential (MMP) is important for evaluating the function of mitochondria, which are particularly vital for the production of energy during sperm movement. The use of flow cytometry, rather than fluorescence microscopy, facilitates high-throughput analysis and enables the quantification of thousands of sperm cells per sample and between the implementation of blind counting and randomization (Holman 2009; Rzymski et al. 2012; Paynter et al. 2014).

We recently evaluated the relationship between the total antioxidant capacity of honeybee workers' hemolymph and IMD exposure (Słowińska et al. 2016). The results revealed that antioxidant protection of honeybees is related to age and may be disturbed by exposure to IMD. In the current study, using the same experimental design, we evaluated whether IMD exposure also affected the quality of drone semen. Although drones do not participate in colony tasks, their life is influenced in a similar manner because they spend their first days inside the colony and are fed with contaminated food and by workers contaminated with IMD. The objective of this study was to evaluate the effects of chronical exposure of honeybee drones to environmental, fieldrealistic concentration (5 ppb) and nonenvironmental concentration (200 ppb) of IMD on sperm concentration, motility, viability, and mitochondrial membrane potential measured in semen obtained from 180 drones originating from 18 colonies. Concentrations of IMD used in our study were aimed to test field-realistic concentrations (5 ppb) as well as potentially lethal concentrations (200 ppb). The latter concentrations was also selected after data of Tapparo et al. (2011), who reported that range high concentrations of IMD (up to $340 \mathrm{ppb}$ ) can be found in guttation drops of corn. The usefulness of absorbance measurements at $600 \mathrm{~nm}$ to estimate sperm concentrations (as established for studies of vertebrates) 
(Ciereszko and Dabrowski 1993) was also evaluated.

\section{MATERIAL AND METHODS}

\subsection{Animals and sampling}

\subsubsection{Honeybee colonies}

The experiment was carried out during the beekeeping season (June-August 2014) and included drones reared in honeybee (Apis mellifera carnica) colonies kept in an apiary near Olsztyn, Poland (lat. 53.745117, long. 20.449361). We used colonies occupying two supers of Wielkopolski hives with $360 \times 260 \mathrm{~mm}$ frames of a type commonly used in Poland. Each colony contained a 2-year-old queen, naturally mated, approximately 40,000 workers, and 20 wax combs.

Eighteen colonies were randomly assigned to three experimental groups. Colonies from group BE (control group) were given food free from imidacloprid (IMD, Bayer Health Care AG, Lerverkusen, Germany), while the food administered to colonies from groups BE- 5 and BE-200 was contaminated with 5 and 200 ppb of IMD, respectively. The bees were fed with sugar syrup (ratio sugar to water 5:3) and pollen pastry made from fresh pollen loads and inverted syrup API-Fortune HF 1575 (ICKO, Bollène, France) in the ratio of 2.5:1.4. The colonies were given $5.5 \mathrm{~kg}$ of the liquid food and $0.3 \mathrm{~kg}$ of the pastry in two portions for a total period of 2.5 months. During the experiment, natural external food was absent. The plants did not produce nectar. Exposure to IMD resulted in its transfer into honeybee bodies as described by Słowińska et al. (2016). We assumed that IMD was transferred also to all individuals in hive, drones included.

\subsubsection{Honeybee males and semen collection}

Queens in all colonies were caged on a drone comb in a 3-comb isolator 4 weeks after the start of feeding. All queens were sisters reared from one mother queen. The queens were released after $24 \mathrm{~h}$, and the combs containing eggs were isolated to prevent further egg laying by the queen. The brooded cells were sealed on the 23rd day after egg laying, the combs with broods were tagged, and then they were placed in isolators with a queen excluder, which were incubated inside the colony. During the next $24 \mathrm{~h}$, the time of drone emergence was checked at 6-h intervals.

The emerged drones were weighed and marked with color, and they were then placed in a lower super in the bee colonies where they remained until the end of the study. The supers were isolated from the bottom and the top with a queen excluder, which prevented the drones but not workers from passing through. The drones stayed in the colonies until they were 15 days old. Three to $4 \mathrm{~h}$ before semen collection, the drones were caught and transported to the laboratory together with workers in cages $(130 \times 115 \times 70 \mathrm{~mm})$ supplied with Apifonda candy. The body weight of drones in all groups was similar $(330.6 \pm 21.5,322.9 \pm 30.3$, and $321.1 \pm 30.7 \mathrm{mg}$, for 0,5 , and $200 \mathrm{ppb}$ of IMD, respectively) and the differences were not statistically significant $(p=0.12$ ).

Semen was collected by provoking the organ to evert by slightly bending the thorax and pressing it with fingers, following the method described by Cobey et al. (2013). Each male provided approximately $1 \mu \mathrm{l}$ of ejaculate.

For each treatment, 10 males from 6 colonies were sampled which resulted in obtaining 180 semen samples from 180 drones originating from 18 colonies.

\subsection{Analytical methods}

\subsubsection{Dilution of semen for analysis}

Semen $(1 \mu \mathrm{l})$ was diluted to a 1:100 ratio in Kiev buffer (Collins 2005). Semen suspensions were further sampled $(10 \mu \mathrm{l})$ for sperm motility analysis $(10 \mu \mathrm{l})$, for sperm concentration estimation, and $(80 \mu \mathrm{l})$ for flow cytometry analysis.

\subsubsection{Sperm motility}

Semen suspensions were further diluted at a 1:3 ratio with Kiev buffer containing $0.5 \%$ bovine serum albumin (final dilution of semen was 1:400). Semen was incubated at $35{ }^{\circ} \mathrm{C}$ for $15 \mathrm{~min}$. Sperm motility was estimated subjectively under the microscope equipped with a heated stage $\left(35^{\circ} \mathrm{C}\right)$ by a single observer in a blinded manner. Live video pictures were taken using an Olympus BX40 microscope (Olympus Optical, Tokyo, Japan) with a $10 \times$ negative phase objective and a Sony CCD black and white camera (SPTM108CE). Spermatozoa were rated as motile if active 
movement (mostly circular with sperm head and tail overlapping) (Wegener et al. 2012) was presented. For each experimental treatment, semen was obtained from 180 males (three IMD concentrations for six colonies, 10 males per dose at each colony). Each individual sample was analyzed in duplicate (about 200 spermatozoa per single analysis).

\subsubsection{Sperm concentration}

Preparation of standard curveSemen obtained from four drones originated for control colonies was used. All semen samples were cream-colored and found at the tip of the genitalia on a bed of white mucus (Rousseau et al. 2015). Ejaculates were first diluted 100 times, then $10 \mu 1$ was sampled and further diluted 17, 20, 25, 30, 35, 40, 50 , and 80 times (final dilutions were 1700, 2000, 2500, $3000,3500,4000,5000$, and 8000, respectively). From these suspensions, $90 \mu \mathrm{l}$ was transferred to UVette disposable cuvettes 50-2000 $\mu$ l (Cat. No.: 952010069, Eppendorf AG, Hamburg Germany), and the absorbance at $600 \mathrm{~nm}$ was measured using a BioPhotometer (Eppendorf, Hamburg, Germany). Sperm suspensions diluted 4000 times were counted using a Bürker chamber. For each drone, counting was made in duplicate. Average number of counted spermatozoa per drone was $91 \pm 11$. Using these results, the sperm concentration was calculated for other dilutions. The results were plotted and a regression equation was calculated.

Measurement of sperm concentration For routine sperm concentration analysis, the samples were diluted 4000 times, the absorbance at $600 \mathrm{~nm}$ was measured and the sperm concentration expressed as $10^{9}$ spermatozoa per microliter was calculated from the regression equation.

\subsubsection{Flow cytometry}

Cell membrane integrity (viability) The sperm $\left(1 \times 10^{6}\right.$ sperm cells per sample) samples were diluted in $100 \mu \mathrm{l}$ of Kiev buffer, and the APC Annexin V apoptosis detection kit (Biolegend) was used according to the manufacturer's instructions to characterize the sample. Briefly, the sperm samples were incubated for $15 \mathrm{~min}$ in the dark at room temperature (RT) in $5 \mu \mathrm{l}$ of APC Annexin V and $5 \mu$ of 7-AAD viability staining solution. Then, $0.4 \mathrm{ml}$ of fresh Kiev buffer was added

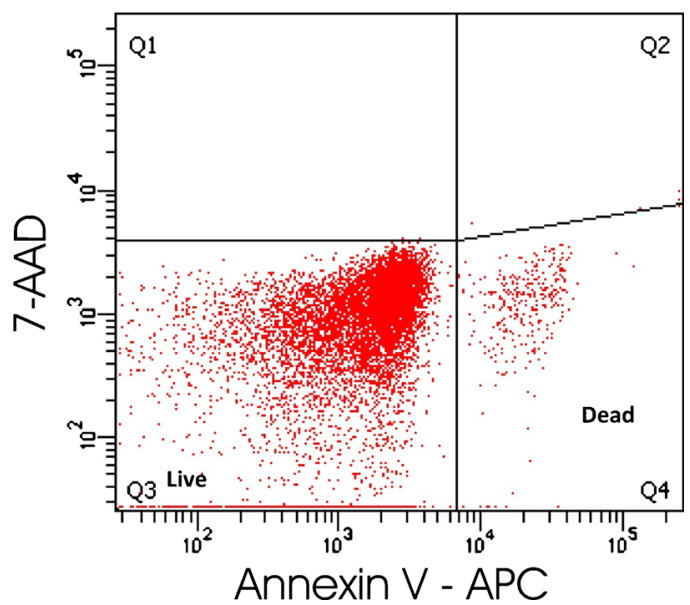

Figure 1. Representative picture for cell membrane integrity (viability) analysis 7-AAD is high affinity DNA dye that enter and stain dead cells, but is impermeable to live cells.

and the samples were analyzed using a flow cytometer FACS Aria II (Becton Dickinson, San Jose, CA). Results were analyzed using DIVA software (Becton Dickinson). A representative picture is shown in Figure 1. This kit was used to evaluate cell membrane integrity (viability) because apoptosis was not observed $(<0.5 \%)$ in the sperm samples.

Mitochondrial membrane potential analysis The sperm samples were diluted to a final concentration of $1.5-2 \times 10^{6}$ sperm cells in $100 \mu$ of Kiev buffer. Then, $5 \mu \mathrm{g} / \mathrm{ml}$ of $\mathrm{Rh} 123$ (Sigma) was added and the sperm suspensions were incubated for $30 \mathrm{~min}$ in the dark at RT. Next, $0.4 \mathrm{ml}$ of Kiev buffer was added and the samples were centrifuged $(400 \times g, 5 \mathrm{~min}, \mathrm{RT})$. Next, the staining medium was removed, the sperm pellets were resuspended in fresh Kiev buffer $(1 \mathrm{ml})$ and counterstained with $5 \mu \mathrm{g} / \mathrm{ml}$ of 7-AAD (Invitrogen). Immediately after the staining, the samples were analyzed using a flow cytometer FACS Aria II (Becton Dickinson, San Jose, CA). The results were analyzed using DIVA software (Becton Dickinson). Approximately 10, 000 spermatozoa were analyzed, and a representative picture is shown in Figure 2.

\subsection{Statistical analysis}

All the results are presented as the mean \pm SD. For statistical analysis, data percentages were transformed by 


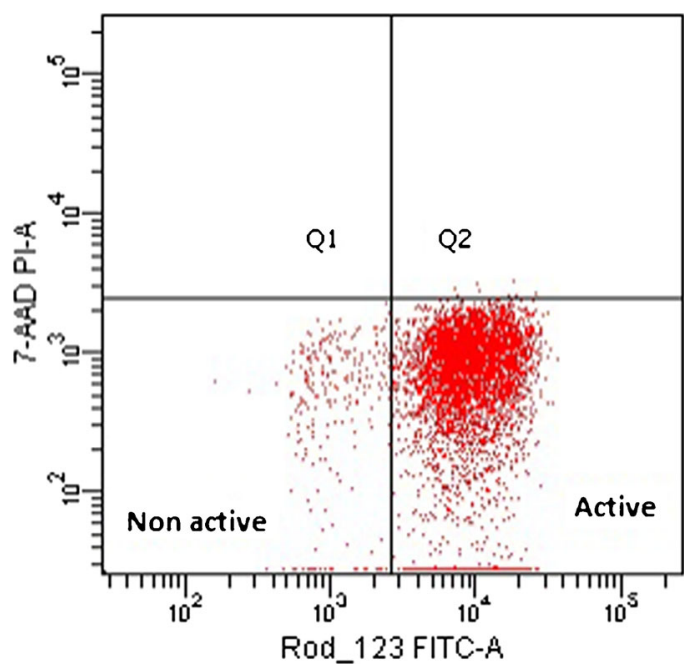

Figure 2. Representative picture for mitochondrial membrane potential analysis Rhodamine 123 selectively accumulates in mitochondria based on the membrane potential.

arcsin square root transformation. The Gaussion distribution of values was confirmed using D'Agostino and Pearson omnibus normality tests. The effect of IMD and colony on semen parameters was analyzed using a twoway ANOVA. Where necessary, the means were separated with a post hoc Tukey's test. Correlation and regression between sperm concentration and absorbance at $600 \mathrm{~nm}$ were calculated using Pearson correlation coefficients. GraphPad Prism (GraphPad Software Inc., San Diego, CA, USA) software was used for statistical calculations.

\section{RESULTS}

\subsection{Regression between sperm concentration and absorbance at $600 \mathrm{~nm}$}

A significant regression between sperm concentration and absorbance at $600 \mathrm{~nm}$ was recorded (Figure 3), and a correlation coefficient was found to be $0.95(p<0.0001)$.

\subsection{Sperm concentration in relation to exposure of honeybees to imidacloprid}

The average sperm concentration in experimental groups varied from 5.9 to $6.0 \times 10^{6}$ spermatozoa per microliter. A significant effect of IMD concentration on sperm concentration was not observed

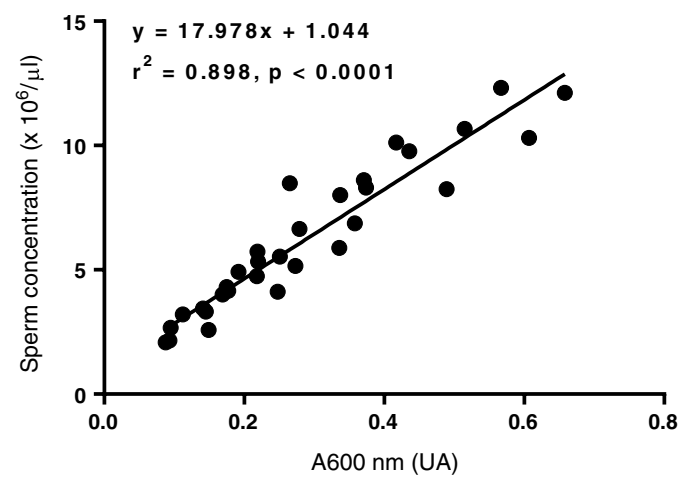

Figure 3. The regression between sperm concentration and absorbance at $600 \mathrm{~nm}(n=32)$. Semen obtained from four drones. Ejaculates were first diluted 100 times, and further diluted 17, 20, 25, 30, 35, 40, 50, and 80 times. Then sperm suspensions were transferred to UVette disposable cuvettes and the absorbance at $600 \mathrm{~nm}$ was measured. Sperm suspensions diluted 4000 times were counted using a Bürker chamber. The results were plotted and a regression equation was calculated

$\left(F_{2,162}=0.19, P=0.83\right)$ (Figure 4a). There was no significant interaction between IMD exposure and colony effects $\left(F_{10,162}=1.31, P=0.23\right)$. However, there was a significant effect of colony on sperm concentration $\left(F_{5,162}=3.38, P=0.006\right)$. This was due to a difference between colony 1 and 4 (Figure 4b).

\subsection{Sperm motility in relation to exposure of honeybees to imidacloprid}

The average sperm motility in experimental groups varied from 69 to $79 \%$. There was a significant effect of imidacloprid on sperm motility $\left(F_{2,162}=10.13, P<0.0001\right)$ between the control and a 200- $\mu$ g concentration of IMD (Figure 5a). A significant difference between colonies was also recorded $\left(F_{5,162}=3.97, P=0.002\right)$ between colonies 1 and 6 (Figure 5b). There was no significant interaction between IMD exposure and colony effects $\left(F_{10,162}=0.65, P=0.77\right)$.

\subsection{Sperm viability in relation to exposure of honeybees to imidacloprid}

The average sperm viability in experimental groups varied from 95 to $99 \%$. There was a 

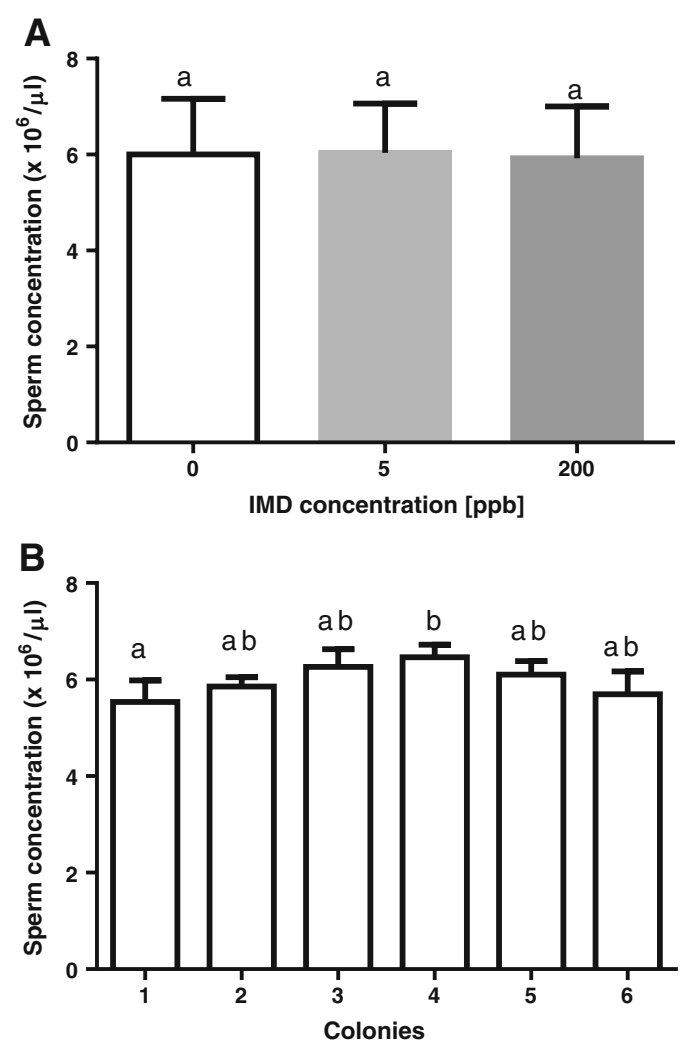

Figure 4. Sperm concentration in relation to exposure of honeybees to imidacloprid (a) and colony (b). Data are presented as mean \pm SD. Male semen was collected by provoking the organ to evert by slightly bending the thorax and pressing it with fingers. For each treatment, 10 males from 6 colonies were sampled which resulted in obtaining 180 semen samples from 180 drones originating from 18 colonies. Data were analyzed using a two-way ANOVA and the means were separated with a post hoc Tukey's test. Sperm concentration bars that share the same letter did not differ significantly $(P<0.05)$

significant effect of imidacloprid on sperm viability $\left(F_{2,162}=9.60, P=0.0001\right)$. A significant difference among colonies was also recorded $\left(F_{5,162}=14.33, P<0.0001\right)$. There was a significant interaction between IMD exposure and colony effects $\left(F_{10,162}=8.18\right.$, $P<0.0001)$. Significant differences were found within colonies for a particular IMD concentration (Figure 6a). The effects of IMD also varied between colonies; for example, no
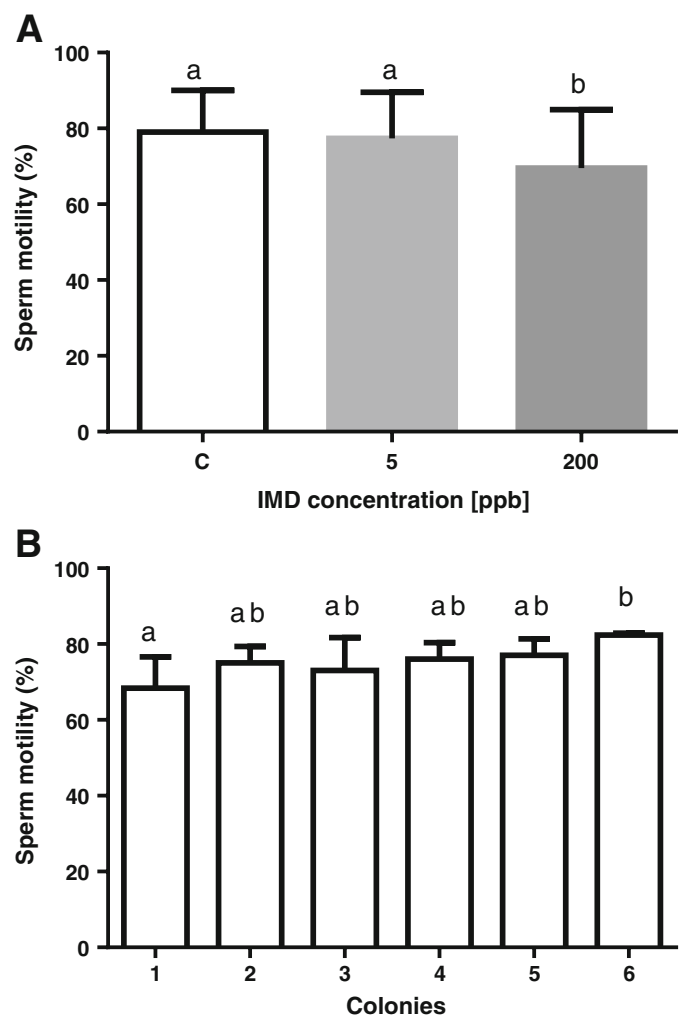

Figure 5. Sperm motility in relation to exposure of honeybees to imidacloprid (a) and colony (b). Sperm motility was estimated subjectively under the microscope equipped with a heated stage $\left(35^{\circ} \mathrm{C}\right)$. Data are presented as mean $\pm \mathrm{SD}$. For each treatment, 10 males from 6 colonies were sampled which resulted in obtaining 180 semen samples from 180 drones originating from 18 colonies. Data were analyzed using a twoway ANOVA and the means were separated with a post hoc Tukey's test. Sperm motility bars that share the same letter did not differ significantly $(P<0.05)$

effects were found in colonies 1 and 6, a decrease in viability was found for colony 2 , and an increase was recorded for colonies 3, 4, and 5 (Figure 6b).

\subsection{Sperm mitochondrial potential in relation to exposure of honeybees to imidacloprid}

The average sperm mitochondrial potential in experimental groups varied from 66 to 

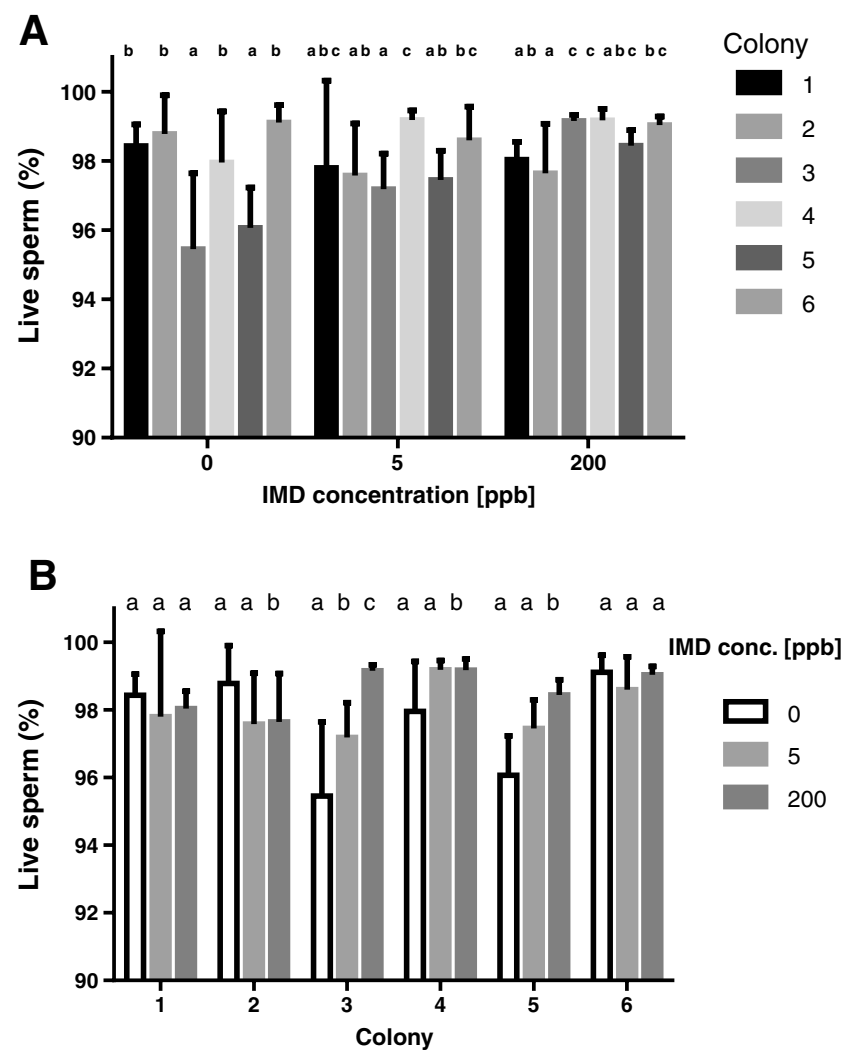

Figure 6. Percentage of live sperm measured by flow cytometry in relation to exposure of honeybees to imidacloprid (a) and colony (b ). Data are presented as mean \pm SD. For each treatment, 10 males from 6 colonies were sampled which resulted in obtaining 180 semen samples from 180 drones originating from 18 colonies. Data were analyzed using a two-way ANOVA and the means were separated with a post hoc Tukey's test. There was a significant interaction between IMD exposure and colony effects $\left(F_{10,162}=8.18, P<0.0001\right)$. Sperm viability bars that share the same letter within the same IMD concentration (a) and colony (b ) did not differ significantly $(P<0.05)$

$93 \%$. There was a significant effect of imidacloprid on sperm mitochondrial potential $\left(F_{2,162}=6.07, P=0.003\right)$. A significant difference within colonies was also recorded $\left(F_{5,162}=12.00, P<0.0001\right)$. There was a significant interaction between IMD exposure and colony effects $\left(F_{10,162}=2.12\right.$, $P=0.03)$.

Significant differences were found within colonies for a particular IMD concentration (Figure 7a). The effects of IMD also varied between colonies; for example, no effects were found for colonies 1 and 6 , a decrease in viability was found for colony 2 , and an increase was recorded for colonies 3,4 , and 5 (Figure 7b).

\section{DISCUSSION}

Most measurements of sperm concentrations in honeybee drones are based on counting the sperm cells under a microscope. This approach is tedious, time consuming, and difficult due to highly variable results that may result from the clumping of spermatozoa and the poor dispersion of spermatozoa in the hemocytometer cells (Collins 2005; Koeniger et al. 2005). Collins (2005) has tested the spectrophotometric method at 260 and $550 \mathrm{~nm}$ and found a significant correlation between sperm counts and absorbance $(r=0.83)$. In our opinion, however, individual points shown on the regression plot in this paper were quite dispersed, and therefore the accuracy of this 

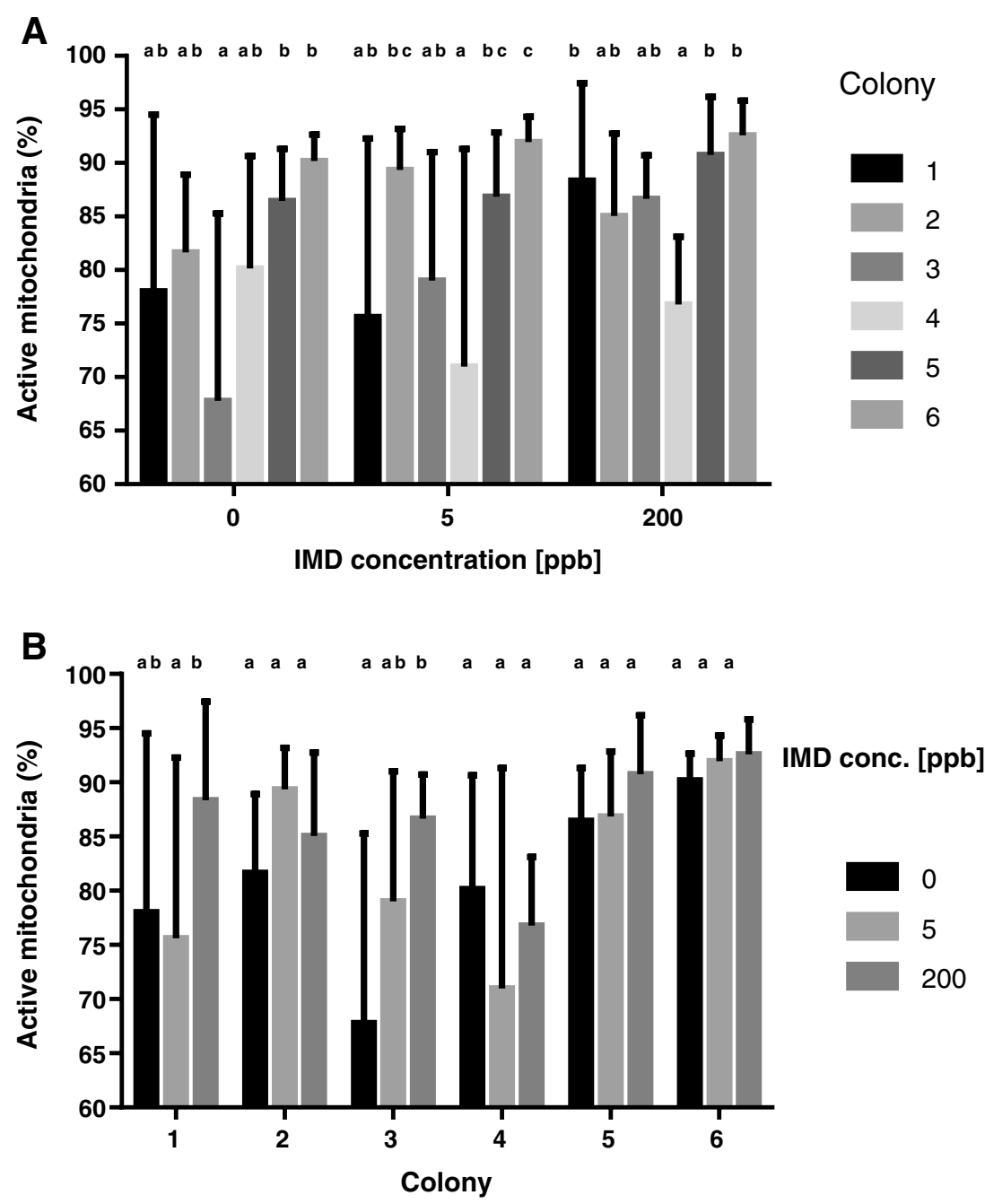

Figure 7. Percentage of active mitochondria in relation to exposure of honeybees to imidacloprid (a) and colony (b). Data are presented as mean \pm SD. For each treatment, 10 males from 6 colonies were sampled which resulted in obtaining 180 semen samples from 180 drones originating from 18 colonies. Data were analyzed using a two-way ANOVA and the means were separated with a post hoc Tukey's test. There was a significant interaction between IMD exposure and colony effects $\left(F_{10,162}=2.12, P=0.03\right)$. Bars that share the same letter within the same IMD concentration (a) and colony (b) did not differ significantly $(P<0.05)$

regression can be improved. Using our modification, we found the sperm concentration estimation data less dispersed, which is confirmed by a high correlation coefficient value $(0.95)$. This result suggests that absorbance at $600 \mathrm{~nm}$ rather than at $550 \mathrm{~nm}$ provides a more accurate estimation of sperm concentrations in bees.

Sperm concentrations stored in spermatheca are recognized as a major component of queen longevity and consequently productivity of the colony and low spermatozoa numbers results in premature supersedure (Cobey 2007). Our results indicated that sperm concentration was not affected by IMD treatment and suggests that drone spermatogenesis was not affected over the period from spermatogenesis to sexual maturity. This is contrary to vertebrates, where morphological and molecular damage in the testis were identified 
after IMD exposure, including changes in testicular architecture and arrested spermatogenesis (Cardone 2015). The latter also indicates an increase in apoptotic processes; however, our results did not show significant numbers of apoptotic spermatozoa $(<0.5 \%)$. Perhaps this discrepancy reflects a significant difference in spermatogenesis and sperm production between insects and mammals (Bishop 1920). The honeybee testes degenerate after hatching, and sperm is stored in the accessory testes (den Boer et al. 2009). In conclusion, spermatogenesis in honeybees seems to be resistant to IMD. The mechanism underlying this resistance deserves further analysis in order to obtain comparative data concerning insect and vertebrate spermatogenesis.

Sperm motility is an important parameter of semen quality. It is estimated that only $2.5 \%$ of inseminated spermatozoa actively migrate into long-term storage (Baer 2005). Wegener et al. (2012) found a strong correlation between sperm motility and indicators of sperm performance in inseminated queens. In our study, we noticed a significant decline in sperm motility only for drones exposed to an extremely high concentration of IMD (200 ppb). This suggests that sperm motility can indeed be affected by neonicotinoids, but such effects seem to be unlikely in environmental conditions where lower concentrations of IMD are recorded. However, sublethal changes in sperm motility cannot be excluded, for example, via cumulative effects of low IMD concentration. Such changes cannot be evaluated by a subjective method for motility estimations as used in our study, rather more sophisticated methods using computer-assisted sperm analysis (Al-Lawati et al. 2009) will be required.

Recent data suggest that despite adequate insemination, the sperm stored in queens may progressively loose viability (Tarpy and Olivarez 2014). A slight reduction $(\sim 10 \%)$ in sperm viability occurs naturally during the second stage of eversion of the endophallus and during the injection of semen into the lateral oviducts of queens (Gençer et al. 2014). A significant variability in the viability of spermatozoa stored in queen spermathecae (with some queens containing less than $20 \%$ viable sperm) has been described in Canada (Rousseau et al. 2015). Tarpy et al. (2012) recorded a 59 to $93 \%$ variability in sperm viability in the spermathecae of commercial honeybee queens. Tarpy and Olivarez (2014) suggested that one of the major reasons for lifespan reductions of queens is a depletion of stored semen, which results in a queen laying unfertilized eggs that develop into drones. It is tempting to speculate that spermatozoa with decreased viability caused by exposure to IMD may have difficulties in coping with the stress and consequently may die prematurely, which would lead to a decrease in the queen's reproductive potential. Recent results of Williams et al. (2015) seem to support this suggestion. The latter indicated a reduced viability of spermatozoa stored in the spermathecae of honeybee queens exposed to IMD. Moreover, recent findings of Sandrock et al. (2014b) who reported $60 \%$ queen supersedure in colonies exposed to two neonicotinoids, thiamethoxam, and clothianidin also suggest long-term effects of neonicotinoids on the quality of spermatozoa inside queen reproductive systems.

To our knowledge, measurements of mitochondrial membrane potential (MMP) was performed for the first time for honeybee drones. This sperm parameter is important for evaluating the function of mitochondria, which are vital for the production of energy during sperm movement. For this reason, a correlation between MMP and sperm motility has been reported for vertebrates (Graham et al. 1990; Espinoza et al. 2009; Paoli et al. 2011). Our results have demonstrated that significant IMD $\times$ colony interactions occur for MMP (similarly to sperm viability). This strongly suggests that IMD toxicity is related to particular colonies, some of which may be more sensitive to IMD than others.

In our study, significant interactions were found between IMD and sperm viability and MMP. For this reason, only conclusions for particular IMD concentration $\times$ colony variants can be formulated. Moreover, all queens were sisters. For this reason, the results of our study can be explained by paternal effects (or by other unexplained defense mechanisms). Our results suggest that honeybee spermatozoa are susceptible to imidacloprid toxicity and this susceptibility is strongly affected by colonies. These results are consistent with the variability of reported IMD 
effects on honeybees. Pisa et al. (2015) reported that the honeybee lethal dose $\left(\mathrm{LD}_{50}\right)$ for imidacloprid varies significantly (by two orders of magnitude) from 5 to $500 \mathrm{ng}$, which can partially be attributed to colonies (but also to individual variability related to mode of contact and differences in environmental conditions). Recently, Stürup et al. (2013) reported that age effects of honeybee drone sperm viability are related to individual colony effects. These authors found a negative correlation between senescence and sperm viability, while a significant interaction between age and colony was also observed (a decline in sperm viability was observed in three of the five colonies). The potential mechanism of differences in sperm viability among colonies may be related to differences in seminal fluid protein abundance (Baer et al. 2012). Perhaps such differences reflect the mechanism of IMD effects on the reproductive potential of drones. It is important to note that under field conditions, there might be no observable effect on queen fecundity since queens mate with up to 20 drones and collect an excess of spermatozoa. In such conditions a sufficient number of viable spermatozoa could be secured. Moreover, under natural mating conditions, there is an extremely high competition of drones, so it is unclear if IMDexposed drones can successfully compete with non-exposed drones. All these precautions have to be taken into account in future studies.

Recently, Williams et al. (2015) demonstrated that the reproductive system of honeybee queens exposed to neonicotinoid pesticides was severely affected morphologically and physiologically. Pesticide-exposed queens developed smaller ovaries (lower number of ovarioles). Moreover, these queens were characterized by a reduced number and viability of spermatozoa stored in their spermathecae. Our data significantly complement these results by demonstrating that male reproduction can be disturbed by neonicotinoids as well. Therefore, it can be suggested that neonicotinoids affect the reproduction capacity of both sexes in honeybees.

In conclusion, our results strongly suggest that neonicotinoids can negatively affect the sperm quality of honeybee drones. Consequently, the exposure of honeybees to neonicotinoids can potentially disturb the reproductive potential of drones and possibly lead to colony failure due poor quality in mating. Colonies are differentially affected by IMD-related changes in sperm quality. Future studies should identify the mechanisms responsible for the variable susceptibility of colonies to IMD effects, to attribute how much of observed effects there was from the colony-level exposure. Knowledge of such mechanisms will be helpful in developing strategies counteracting the negative effects of IMD on honeybee reproduction. Moreover, due to large variation in IMDresponse among colony future experiments should focus on feeding individual drones with IMD.

\section{ACKNOWLEDGMENTS}

This work was funded by the Institute of Animal Reproduction and Food Research and the University of Warmia and Mazury in Olsztyn. We would like to thank Dr. Gabriel Bodek from the In Vitro Laboratory for performing flow cytometry analyses.

\section{OPEN ACCESS}

This article is distributed under the terms of the Creative Commons Attribution 4.0 International License (http://creativecommons.org/licenses/by/4.0/), which permits unrestricted use, distribution, and reproduction in any medium, provided you give appropriate credit to the original author(s) and the source, provide a link to the Creative Commons license, and indicate if changes were made.

Paramètres du sperme des mâles d'abeille exposés à l'imidaclopride Apis mellifera / néonicotinoïde / spermatozoïde / motilité / viabilité

Parameter des Drohnenspermas der Honigbiene nach Imidacloprid-Exposition Apis mellifera / Imidacloprid / Spermatozoa / Beweglichkeit / Viabilität

\section{REFERENCES}

Al-Lawati, H., Kamp, G., Bienefeld, K. (2009) Characteristics of the spermathecal contents of old and young honeybee queens. J. Insect Physiol. 55(2), 116-121

Baer, B. (2005) Sexual selection in Apis bees. Apidologie 36(2), 187-200 
Baer, B., Zareie, R., Paynter, E., Poland, V., Millar, A.H. (2012) Seminal fluid proteins differ in abundance between genetic lineages of honeybees. J. Proteom. 75(18), 5646-5653

Bal, R., Naziroğlu, M., Türk, G., Yilmaz, O., Kuloğlu, T., et al. (2012) Insecticide imidacloprid induces morphological and DNA damage through oxidative toxicity on the reproductive organs of developing male rats. Cell Bioch. Funct. 30 (6), 492-499.

Ben Abdelkader, F., Kairo, G., Tchamitchian, S., Cousin, M., Senechal, J., et al. (2014) Semen quality of honey bee drones maintained from emergence to sexual maturity under laboratory, semi-field and field conditions. Apidologie 45 (2), 215-223

Bishop, G.H. (1920) Fertilization in the honey-bee. I. The male sexual organs: Their histological structure and physiological functioning. J. Exp. Zool. 31 (2), 224-265

Blacquière, T., Smagghe, G., Van Gestel, C.A.M., Mommaerts, V. (2012) Neonicotinoids in bees: a review on concentrations, side-effects and risk assessment. Ecotoxicology 21 (4), 973-992

Cardone, A. (2015) Imidacloprid induces morphological and molecular damages on testis of lizard (Podarcis sicula ). Ecotoxicology 24 (1), 94-105

Ciereszko, A., Dabrowski, K. (1993) Estimation of sperm concentration of rainbow trout, whitefish and yellow perch by spectrophotometric technique. Aquaculture 109, 367-373

Cobey, S.W. (2007) Comparison studies of instrumentally inseminated and naturally mated honey bee queens and factors affecting their performance. Apidologie 38 (4), $390-410$

Cobey, S.W., Tarpy, D.R., Woyke, J. (2013) Standard methods for instrumental insemination of Apis mellifera queens. J. Apic. Res. 52 (4), 1-18

Collins, A.M. (2005) Insemination of honey bee, Apis mellifera, queens with non-frozen stored semen: sperm concentration measured with a spectrophotometer. J. Apic. Res. 44 (4), 141-145

Cresswell, J.E. (2011) A meta-analysis of experiments testing the effects of a neonicotinoid insecticide (imidacloprid) on honey bees. Ecotoxicology, 20 (1), 149-157

Cresswell, J. (2014) On the natural history of neonicotinoids and bees. Funct. Ecol. 28(6), 1311-1312

Den Boer, S.P.A., Boomsma, J.J., Baer, B. (2009) Honey bee males and queens use glandular secretions to enhance sperm viability before and after storage. J. Insect Physiol. 55 (6), 538-543

Dively, G.P., Embrey, M.S., Kamel, A., Hawthorne, D.J., Pettis, J.S. (2015) Assessment of chronic sublethal effects of imidacloprid on honey bee colony health. PLoS ONE, 10 (3), e0118748

Espinoza, J.A., Schulz, M.A., Sanchez, R., Villegas, J.V. (2009) Integrity of mitochondrial membrane potential reflects human sperm quality. Andrologia 41 (1), 5154
Gençer, H.V., Kahya, Y., Woyke, J. (2014) Why the viability of spermatozoa diminishes in the honeybee (Apis mellifera ) within short time during natural mating and preparation for instrumental insemination. Apidologie 45 (6), 757-770

Goulson, D (2013) An overview of the environmental risks posed by neonicotinoid insecticides. J. Appl. Ecol. 50 (4), 977-987 DOI: 10.1111/1365-2664.12111

Graham, J.K., Kunze, E., Hammerstedt, R.H. (1990) Analysis of sperm cell viability, acrosomal integrity, and mitochondrial-function using flow-cytometry. Biol. Reprod. 43 (1), 55-64.

Holman, L. (2009) Sperm viability staining in ecology and evolution: potential pitfalls. Behav. Ecol. Sociobiol. 63 (11), 1679-1688

Hunter, F.M., Birkhead, T.R. (2002) Sperm viability and sperm competition in insects. Curr. Biol. 12: 121-123

Koeniger, G., Koeniger, N., Tingek, S., Phiancharoen, M. (2005) Variance in spermatozoa number among Apis dorsata drones and among Apis mellifera drones. Apidologie, 36 (2), 279-284

Lonare, M., Kumar, M., Raut, S., More, A., Doltade, S., et al. (2015) Evaluation of ameliorative effect of curcumin on imidacloprid-induced male reproductive toxicity in Wistar rats. Environ. Toxicol.: 10.1002/ tox. 22132

Lopez-Antia, A., Ortiz-Santaliestra, M.E., Mougeot, F., Mateo, R. (2015) Imidacloprid-treated seed ingestion has lethal effect on adult partridges and reduces both breeding investment and offspring immunity. Environ. Res. 136, 97-107

Paoli, D., Gallo, M., Rizzo, F., Baldi, E., Francavilla, S., et al. (2011) Mitochondrial membrane potential profile and its correlation with increasing sperm motility. Fertil. Steril. 95 (7), 2315-2319.

Paynter, E., Baer-Imhoof, B., Linden, M., Lee-Pullen, T., Heel, K., et al., (2014) Flow cytometry as a rapid and reliable method to quantify sperm viability in the honeybee Apis mellifera . Cytometry Part A 85 (5), 463-472

Pisa, L.W., Amaral-Rogers, V., Belzunces, L.P., Bonmatin, J.M., Downs, C.A., et al. (2015) Effects of neonicotinoids and fipronil on non-target invertebrates. Environ. Sci. Pollut. Res. 22 (1), 68-102

Pohorecka, K., Skubida, P., Miszczak, A., Semkiw, P., Sikorski, P., et al. (2012) Residues of neonicotinoid insecticides in bee collected plant materials from oilseed rape crops and their effect on bee colonies. J. Apic. Sci., 56 (2), 115-134 DOI: 10.2478/v10289012-0029-3

Rhodes, J.W., Harden, S., Spooner-Hart, R., Anderson, D.L., Wheen, G. (2011) Effects of age, season and genetics on semen and sperm production in Apis mellifera drones. Apidologie 42 (1), 29-38

Rousseau, A., Fournier, V., Giovenazzo, P. (2015) Apis mellifera (hymenoptera: Apidae) drone sperm quality in relation to age, genetic line, and time of breeding. Can. Entomol. 147 (6):1-10 
Ruttner, F., Koeniger, G. (1971) Die Füllung der Spermatheka der Bienenkönigin. Aktive Wanderung oder passiver Transport der Spermatozoen? Z. Vgl. Physiol. 72 , 411-422

Rzymski, P., Langowska, A., Fliszkiewicz, M., Poniedziałek, B., Karczewski, J., et al. (2012) Flow cytometry as an estimation tool for honey bee sperm viability. Theriogenology 77 (8), 1642-1647

Sandrock, C., Tanadini, L.G., Pettis, J.S., Biesmeijer, J.C., Potts, S.G., et al. (2014a) Sublethal neonicotinoid insecticide exposure reduces solitary bee reproductive success. Agricult. Forest Entomol. 16 (2), 119-128

Sandrock, C., Tanadini, M., Tanadini, L.G., Fauser-Misslin, A., Potts, S.G., et al. (2014b) Impact of chronic neonicotinoid exposure on honeybee colony performance and queen supersedure. PLoS ONE 9 (8), e103592

Severson, D.W., Erickson, E.H. (1989) Seasonal constraints on mating and insemination of queen honey bee in a continental climate. Apidologie 20 (1), 21-27

Simon-Delso, N., Amaral-Rogers, V., Belzunces, L.P., Bonmatin, J.M., Chagnon, M., et al. (2015) Systemic insecticides (neonicotinoids and fipronil): trends, uses, mode of action and metabolites. Environ. Sci. Pollut. Res. 22 (1), 5-34

Słowińska, M., Nynca, J., Wilde, J., Bąk, B., Siuda, M., et al. (2016) Total antioxidant capacity of honeybee haemolymph in relation to age and exposure to pesticide, and comparison to antioxidant capacity of seminal plasma. Apidologie 47 (2), 227-236

Stürup, M., Baer-Imhoof, B., Nash, D.R., Boomsma, J.J., Baer, B. (2013) When every sperm counts: factors affecting male fertility in the honeybee Apis mellifera . Behav. Ecol. 24 (5), 1192-1198
Tapparo, A., Giorio, C., Marzaro, M., Marton, D., Soldà L, et al. (2011) Rapid analysis of neonicotinoid insecticides in guttation drops of corn seedlings obtained from coated seeds. J. Environ. Monit. 13 (6), 1564-8

Tarpy, D.R., Olivarez, R. (2014) Measuring sperm viability over time in honey bee queens to determine patterns in stored-sperm and queen longevity. J. Apicult. Res. 53 (4), 493-495

Tarpy, D.R., Keller, J.J., Caren, J.R., Delaney, D.A. (2012) Assessing the mating 'health' of commercial honey bee queens. J. Econ. Entomol. 105 (1), 20-25

Tofilski, A., Chuda-Mickiewicz, B., Czekońska, K., Chorbiński, P. (2012) Flow cytometry evidence about sperm competition in honey bee (Apis mellifera). Apidologie 43 (1), 63-70

van der Sluijs, J.P., Simon-Delso, N., Goulson, D., Maxim, L., Bonmatin, J.-M., et al. (2013) Neonicotinoids, bee disorders and the sustainability of pollinator services. Curr. Opin. Env. Sust. 5 (3-4), 293-305

Wegener, J., May, T., Knollmann, U., Kamp, G., Müller, K., et al. (2012) In vivo validation of in vitro quality tests for cryopreserved honey bee semen. Cryobiology 65 (2), 126-131

Whitehorn, P.R., O'Connor, S., Wackers, F.L., Goulson, D. (2012) Neonicotinoid pesticide reduces bumble bee colony growth and queen production. Science 336 (6079), 351-352

Williams, G.R., Troxler, A., Retschnig, G., Roth, K., Yanez, O., et al. (2015) Neonicotinoid pesticides severely affect honey bee queens. Sci. Rep. 5, 14621 DOI: $10.1038 /$ srep14621. 